\title{
Fast neutron measurements with solid state detectors at pulsed spallation sources
}

\author{
C. Cazzaniga, M. Rebai, R. García Alía, P. Fernandez-Martinez, \\ M. Cecchetto, M. Kastriotou, M. Tardocchi, C. D. Frost
}

\section{Published version information}

Citation: C Cazzaniga et al. 'Fast neutron measurements with solid state detectors at pulsed spallation sources.' Journal of Neutron Research, vol. 22, no. 2-3 (2020): 345-352. Is in proceedings of: 23rd Meeting of the International Collaboration on Advanced Neutron Sources (ICANS-XXIII), Chattanooga, Tennessee, United States, 13-18 Oct 2019.

DOI: $\underline{10.3233 / J N R-190141}$

The final publication is available at IOS Press through DOI above.

This version is made available in accordance with publisher policies. Please cite only the published version using the reference above. This is the citation assigned by the publisher at the time of issuing the AAM. Please check the publisher's website for any updates. 


\title{
1 Fast neutron measurements with solid state 2 detectors at pulsed spallation sources
}

\author{
3 C. Cazzaniga ${ }^{\mathrm{a}, 1}$, M. Rebai ${ }^{\mathrm{b}}$, R. García Alía ${ }^{\mathrm{c}}, \mathrm{P}$. Fernandez-Martinez ${ }^{\mathrm{c}}$, M. Cecchetto ${ }^{\mathrm{c}}, \mathrm{M}$.

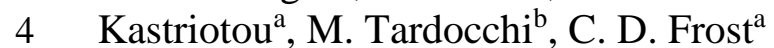 \\ 5 \\ ${ }^{a}$ ISIS Facility, STFC, Rutherford Appleton Laboratory, Didcot OX110 QX, UK \\ ${ }^{\mathrm{b}}$ Istituto di Fisica del Plasma, Consiglio Nazionale delle Ricerche, Milano, Italy \\ ${ }^{\mathrm{c}} \mathrm{CERN}, \mathrm{CH}-1211$, Genève, Switzerland
}

\begin{abstract}
Fast neutron measurements have been performed with silicon and diamond detectors at nTOF, ChipIr, and CHARM facilities. The detectors have been used in pulse mode; the deposited energy and time stamp is measured event by event for each signal above threshold. The pulsed nature of the spallation sources gives high instantaneous counting rates that dictate the use of a fast electronic chain including a current preamplifier and digital acquisition. The energy-dependent response functions of these detectors to fast neutrons can be extracted from nTOF data using time-of-flight to provide energy tagging. These measured response functions can then be used both to benchmark Monte Carlo simulations of the response functions and to interpret the measurements at ChipIR and CHARM, facilities used for the irradiation of microelectronics, where time-of-flight energy measurement at these neutron energies is not possible.
\end{abstract}

Keywords. Neutron measurements, silicon detector, diamond detector, spallation source

\section{Introduction}

Solid state detectors, in particular diamond and silicon detectors, are useful for the measurement of fast neutrons at spallation sources [1-2]. They can be useful both for counting, i.e. flux measurements, and for spectroscopy. Silicon and diamond can be used for measurement of thermal neutrons when a converting isotope is added, typically a layer containing ${ }^{6} \mathrm{Li}$ or ${ }^{10} \mathrm{~B}$ doping [3-4]. For fast neutrons $(\mathrm{MeV}$ range and above), on the other hand, the required charge deposition is provided by the recoil of silicon and carbon nuclei, respectively, and by various nuclear reactions, like $(n, \alpha),(n, p)$, etc., that have negative $Q$ values and energy thresholds above $4 \mathrm{MeV}$ [5].

The main advantages of these detectors are compact dimensions, fast signals (tens of ns) due to the high mobility of electrons and holes in the materials, and low sensitivity to gamma background thanks to pulse height discrimination capability [6-8]. All these properties are important in particular when operating at high instantaneous count rates, as it is often the case at pulsed spallation sources [9-12].

Diamond is a more interesting solution when operating in harsh environments, like high fluences ( > $10^{16} \mathrm{n} / \mathrm{cm}^{2}$ ) [13] or high temperature [14]. On the other hand, it suffers from polarization effects that are

\footnotetext{
${ }^{1}$ Corresponding author. E-mail: carlo.cazzaniga@stfc.ac.uk.
} 
induced by trapping of charge in impurities and defects of the crystal, while silicon is intrinsically more stable [15-16].

Fast neutrons are often the source of background in neutron diffraction experiments, where thermal neutrons are used. The measurements of fast neutrons in the range $>10 \mathrm{MeV}$ at spallation sources is today motivated in good part by the application of fast neutron beamlines for the irradiation of microelectronics. Test engineers can use these facilities to study single event effects on devices and systems and scale the expected error rate to the application environment, typically ground-level, avionics, space and accelerators [17-19].

In this work we used the nTOF facility at CERN [20], where the short primary pulse width and long flight paths enable fast and high energy neutron time-of-flight, to measure the response functions of the detectors. The response functions can benchmark Monte Carlo simulations that have been used to interpret the results at other facilities, in particular in this work the ChipIr facility at ISIS [21] and the CHARM facility [22] at CERN.

New fast neutron beamlines are now proposed at the SNS [23] and CSNS [24]. The present work can be useful in this effort. Furthermore, development in detection of fast neutrons is important in other applications, like next generation nuclear fusion experimental devices [25-27].

\section{Experimental}

\subsection{The facilities}

ChipIr, for Chip Irradiation, is a new beamline at Target Station 2 of the ISIS spallation neutron source of the Rutherford Appleton Laboratory, where the $800 \mathrm{MeV}$ proton beam is collided on a tungsten target. The ChipIr beamline is 14 meters long, and has been designed to extract a fast atmospheric-like neutron spectrum. The neutron flux of about $5 \cdot 10^{6} \mathrm{~s}^{-1} \mathrm{~cm}^{-2}$ [30] with $\mathrm{E}>10 \mathrm{MeV}$ is deemed to be ideal for single event effect testing of microelectronics. The proton beam typically runs $40 \mu \mathrm{A}$ on target and it is pulsed at $10 \mathrm{~Hz}$. Every pulse is composed of two bunches, about $70 \mathrm{~ns}$ wide, $360 \mathrm{~ns}$ apart. This time structure from the accelerator, with the 14 meter flight path, does not allow for time-of-flight measurements in the fast neutron regime.

CHARM, for CERN High energy AcceleRator Mixed field, uses an extraction line of the CERN Proton Synchrotron (PS) accelerator that delivers $24 \mathrm{GeV}$ protons to a copper target [22]. Mixed fields of high energy hadrons are produced (mainly neutrons, pions and protons), and irradiation positions can be found at different angles. We used the G0 position that is about $90 \mathrm{deg}$ respect to the proton beam and dominated by neutrons. Here again the time structure from the accelerator, together with the flight path length, does not allow for time-of-flight spectrometry, as every extraction is $350 \mathrm{~ms}$ long, with a spill periodicity of 5 to 45 seconds, and the flight path in the order of meters.

The neutron Time-Of-Flight facility at CERN (nTOF) is a 200 meters long beamline designed mainly for cross section measurements. Neutrons are produced by spallation of $24 \mathrm{GeV}$ protons from the PS accelerator on a lead target (using a different extraction line than CHARM). The pulses are very short (about $4 \mathrm{~ns}$ ) in order to allow good energy resolution in ToF for epithermal and fast neutrons over the 200 $\mathrm{m}$ flight path length.

The energy spectra of the three facilities are presented in Figure 1 for comparison [22,29-31]. It is interesting to say that, due to the very different characteristics of the facilities the spectra are measured with different techniques, including the unfolding of activation foils [29-30] and fission chambers [31]. 
The detectors are a $2 \mathrm{~mm} \times 2 \mathrm{~mm} \times 140 \mu \mathrm{m}$ silicon diode and a $2 \mathrm{~mm}$ x $2 \mathrm{~mm}$ x $300 \mu \mathrm{m}$ diamond detector. The silicon detector is a totally depleted $\mathrm{p}-\mathrm{n}$ junction diode manufactured by Micron Semiconductors Ltd. To be used as a detector it is reverse biased by applying $40 \mathrm{~V}$. The diamond is a solid-state ionization chamber, and being an insulator it can be biased in both directions. During the test $+240 \mathrm{~V}$ were applied. Diamond of the required purity and electronic grade are produced by chemical vapour deposition by Element Six. The geometry of the detectors is square and features a ceramic PCB and a metal housing

The pulsed nature of the spallation sources gives very high instantaneous counting rates that dictate the use of a fast electronic chain, with a current preamplifier and digital acquisition. The low noise current preamplifier is a Cividec $\mathrm{C} 1$ with an analog bandwidth of $2 \mathrm{GHz}$ and a $20 \mathrm{~dB}$ gain. The digitizer is a CAEN model, 1 Gsample/sec, 10 bits. All the waveforms are recorded using the digitizer in oscilloscope mode, triggering on the accelerator extraction signal. The off-line analysis extracts for every neutron interaction the time-stamp and the pulse area, so that time of flight and deposited energy spectra can be determined. The time-stamp is defined with the crossing of a threshold level that is set above the electronic noise. Due to the very fast rise time and $1 \mathrm{Gsample/sec} \mathrm{sampling} \mathrm{rate,} \mathrm{we} \mathrm{expect} \mathrm{the} \mathrm{error} \mathrm{on}$ the time-stamp to be in the order of $1 \mathrm{~ns}$. A schematic of the setup is presented in Figure 2.

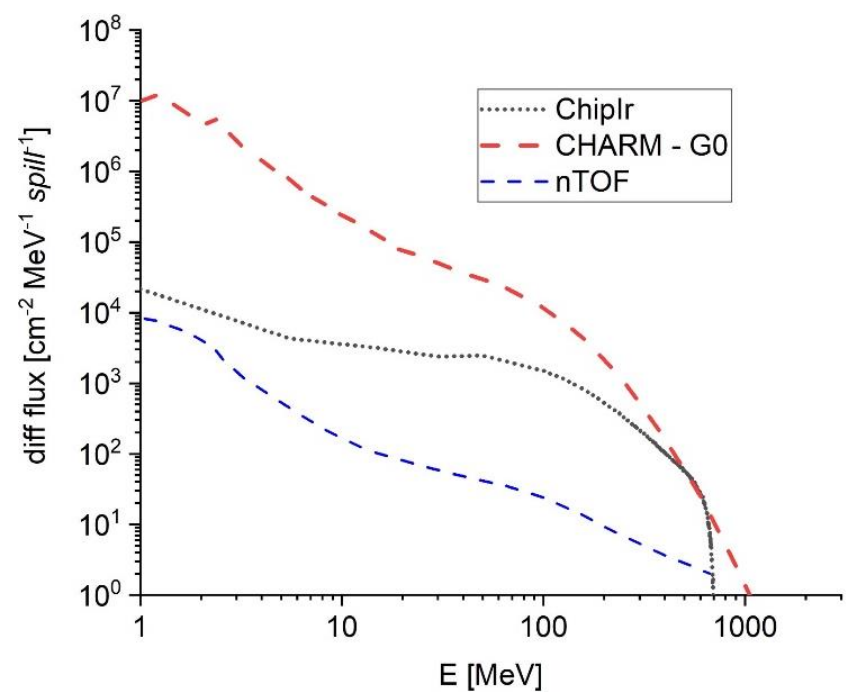

Figure 1. The differential neutron energy spectra of the three facilities as from literature (ChipIr [29, 30], CHARM [29], nTOF [31,32]). The flux is specified at the standard sample position of each beamline. 


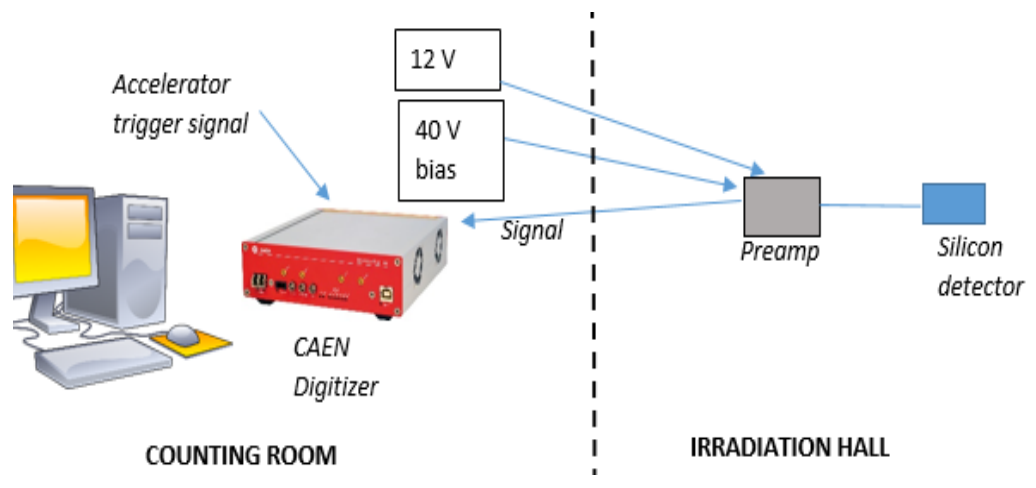

Figure 2. Schematics of the experimental setup of solid-state detectors operated at spallation facilities.

\section{Measurements of fast neutrons response functions at nTOF}

The data analysis at nTOF is based on the measurement, for each pulse, of deposited energy and time of flight. As all the data is acquired in oscilloscope mode, this analysis is done offline by a dedicated software. Examples of acquired waveforms are shown in Figure 3.

The software discriminates all the pulses above a voltage threshold (typical value is $5 \mathrm{mV}$ above the filtered baseline), and calculates the area of each pulse subtracting the baseline. As it can be seen in Figure 3 , there are typically multiple pulses for every acquired waveform. The timescale, here in ns, is the delay from the $\mathrm{T}_{\text {start }}$ signal from the nTOF extraction line from the PS accelerator. The ToF can be calibrated by subtracting the constant ToF of $\gamma$-rays, which always arrive as a burst, at about $13050 \mathrm{~ns}$ in Figure 3 . Figure 4 shows the resulting measured ToF spectra for silicon and diamond, as it is obtained when a histogram with all the events above threshold is built.

The kinetic energy of a neutron $\left(E_{n}\right)$ can therefore be calculated using the relativistic formula as in eq. 1 , where $m_{0}$ is the rest mass of the neutron and $\mathrm{L}$ is the flight path of $200 \mathrm{~m}$.

$$
E_{n}=m_{0} c^{2}\left(\frac{1}{\sqrt{1-\frac{L^{2}}{c^{2} T o F^{2}}}}-1\right)
$$

The deposited energy $\left(E_{d e p}\right)$ is proportional to the area of the signal as in eq. 2, where $E_{e h}$ is the energy to produce a pair of electron-hole, $G$ is the gain of the preamplifier, $e$ is the fundamental charge, and $R$ is $50 \Omega$.

$$
E_{d e p}=\frac{E_{e h}}{G \cdot e \cdot R} \int V(t) d t
$$




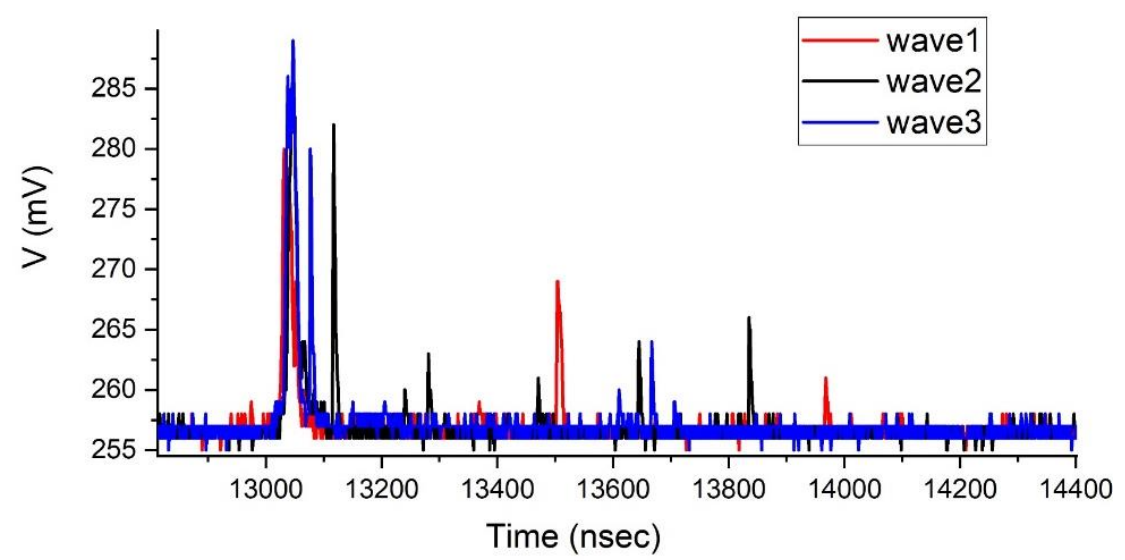

Figure 3. Example of waveforms recorded by the silicon detector at nTOF. For example, the first waveform, in red, shows two neutron events following the gamma burst at around $13050 \mathrm{~ns}$.

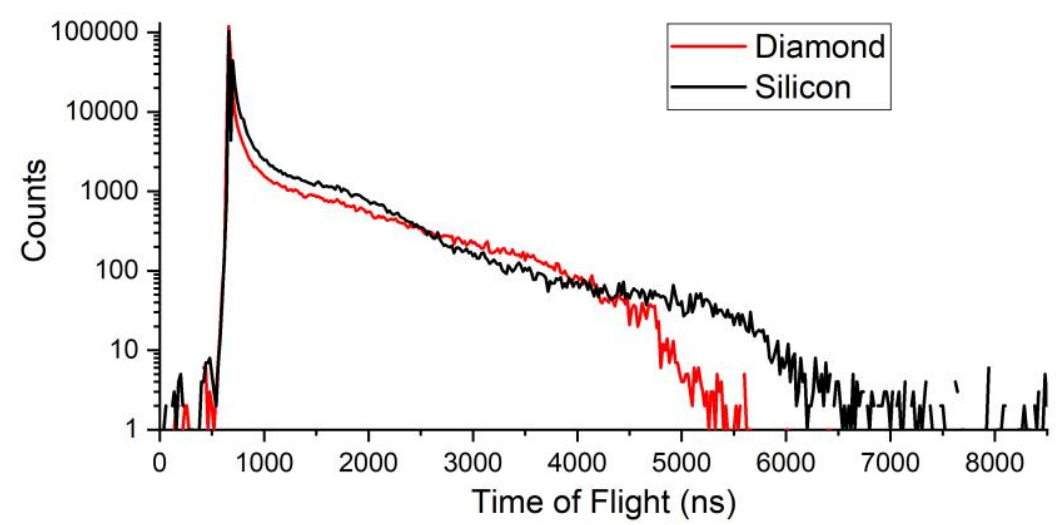

Figure 4. Time of flight counting spectra measured at nTOF, where the short pulse width and long flight path allow us to perform fast neutron spectrometry with time-of-flight.

The measured response functions shown in Figure 5 are obtained building the histogram of the deposited energy for selected values of $E_{n} \pm 0.1 E_{n}$. The values have been selected as representative examples in the considered energy range, but from the data one can choose any given value of interest. In this case, an accuracy on $\mathrm{E}_{\mathrm{n}}$ of $10 \%$ has been selected, but one can chose to have a better accuracy at the price of poorer statistics.

Finally, the measurements are compared to Monte Carlo calculation of the response functions, using a model developed with FLUKA [13]. Further work is being done to extend the simulations using a new version of FLUKA and GEANT4. 


\section{Silicon detector}
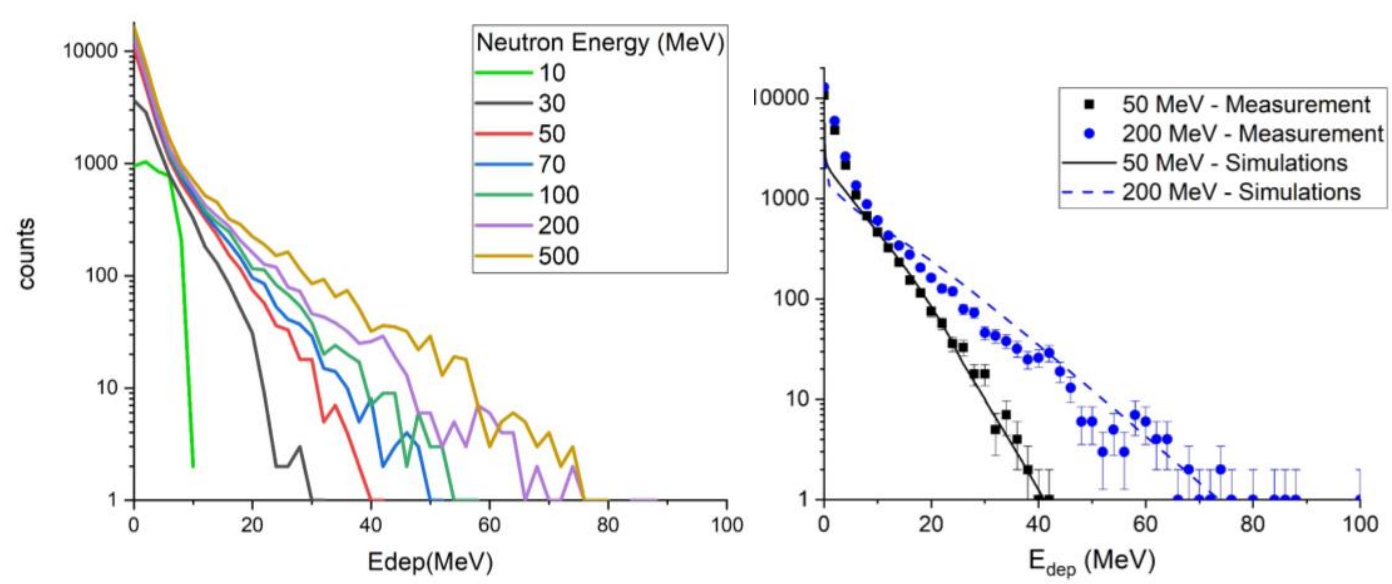

Diamond detector
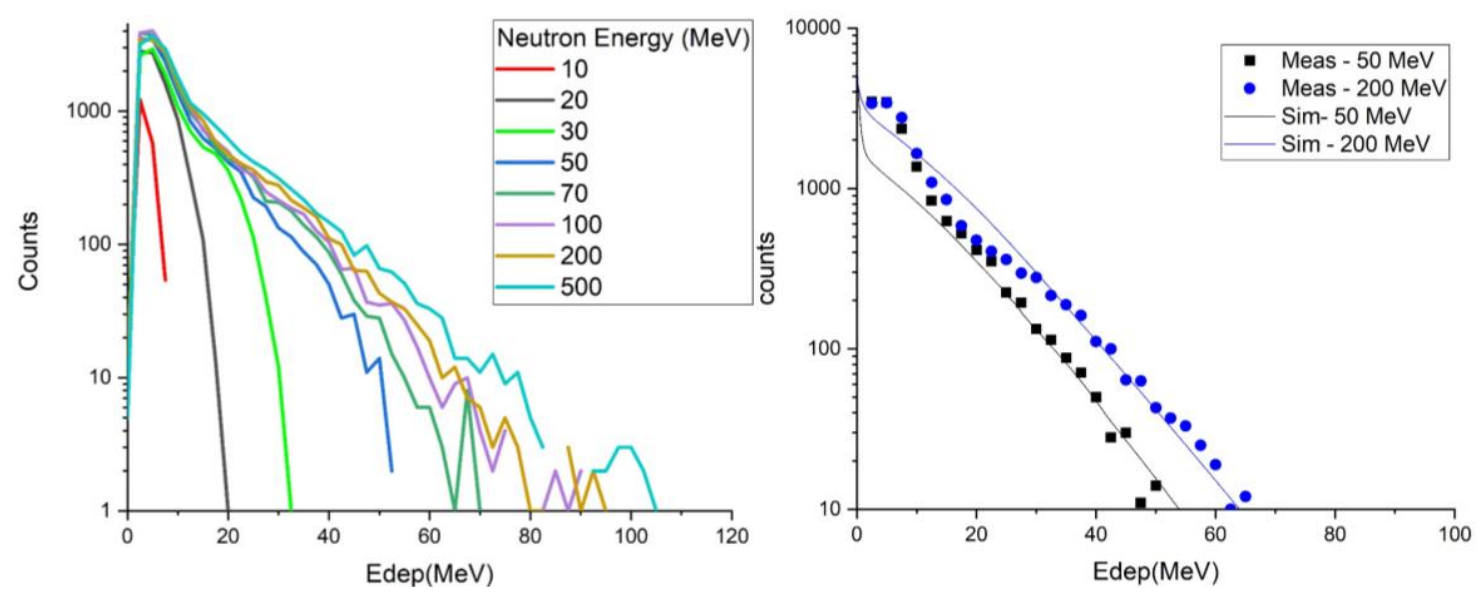

Figure 5. Fast neutron response functions as measured at nTOF (left). The right-hand panels show a subset of those measured response functions compared to FLUKA simulations. The upper panels are for the silicon detector and the lower panels are for the diamond detector.

\section{Measurements at electronics irradiation facilities}

These detectors, now with the response functions described above, have then been used to characterise CHARM and ChipIr, the two facilities dedicated to the irradiation of microelectronics [28]. The time characteristics of the extraction and the short flight paths do not allow here for ToF measurements. The deposited energy spectra have been measured and are reported in Figure 6. The simulations, based on the same FLUKA model, that have been benchmarked with nTOF data for mono-energetic neutrons, have

11 been repeated using as input the spectra provided by the facilities (see Figure 1). 

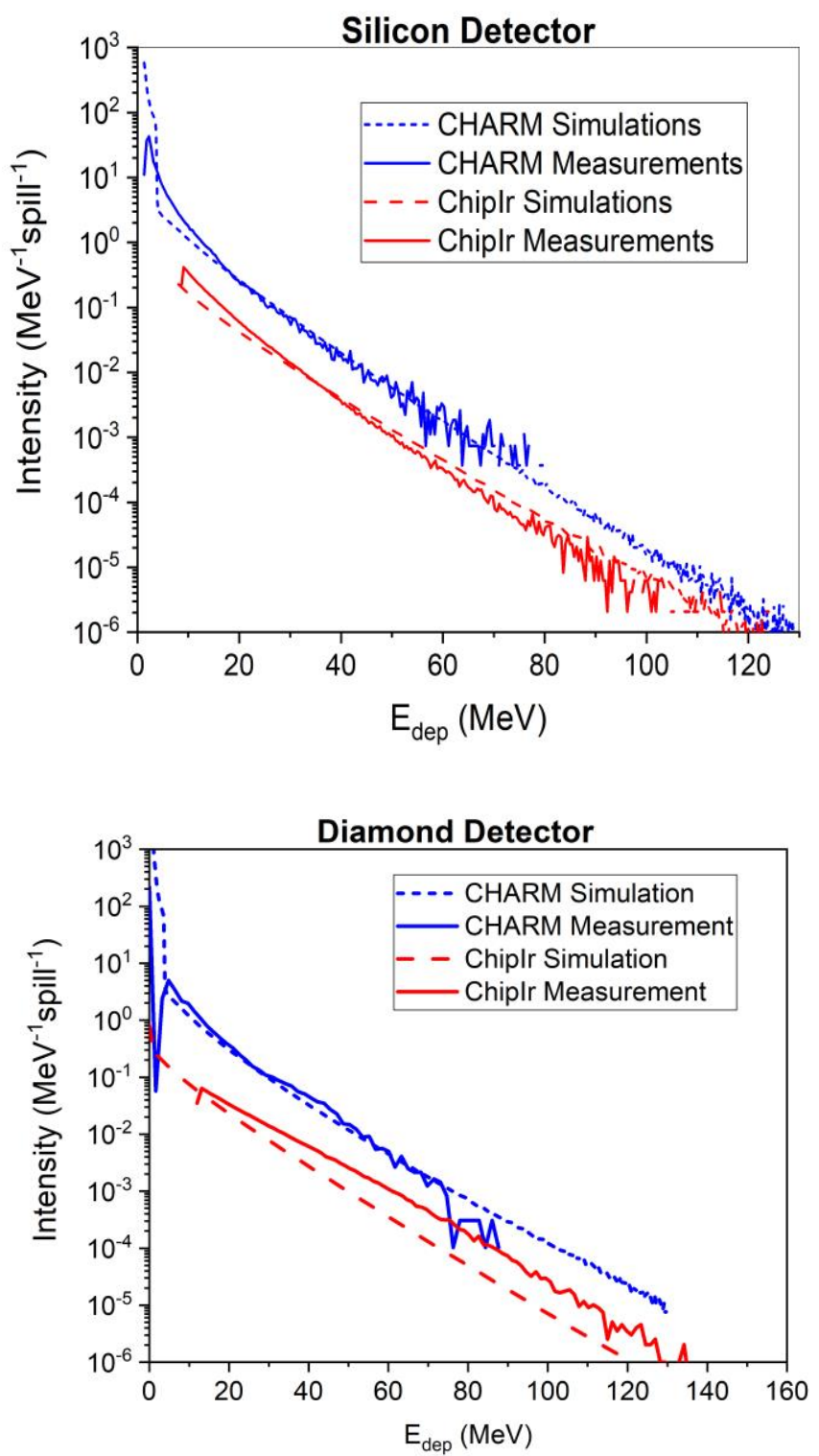

Figure 6. Deposited energy spectra measured at ChipIr and CHARM, and compared to FLUKA simulations, using a silicon detector (top) and diamond detector (bottom).

The result is a highly satisfactory agreement between the simulated and measured energy deposition distributions. In particular, at CHARM both for silicon and diamond, and at ChipIr for silicon, the difference between measurement and simulation is less than $10 \%$ in the region $20 \mathrm{MeV}<\mathrm{E}_{\text {dep }}<80 \mathrm{MeV}$. This gives confidence in the facilities characterization and in the same modelling tools that are often used 10 to simulate single event effects rate in microelectronics in complex radiation environments. We note that 11 diamond data at ChipIr deviate from the predicted curve more than measured data for Si detector. 12 Polarization effects are a possible cause of degradation. A second possibility is that, due to the larger size $13(300 \mu \mathrm{m}$ vs. $140 \mu \mathrm{m})$ the diamond detector suffers more of signal pile-up effect, that can also induce a degradation in the measured spectrum. Both effects are less relevant at CHARM, where, due to the 
different structure of the pulse, the instantaneous flux is lower (although the intensity per spill is higher). Further investigation is needed to better understand and possibly reduce this discrepancy.

Finally, we consider the use of these detectors as beam monitors and focus on their stability. The deposited energy time trace is measured with both detectors at CHARM and ChipIR and presented in Figure 7. For CHARM the number of spills is used as time variable because the spills are much more sparse (one every about $30 \mathrm{~s}$, against $10 \mathrm{~Hz}$ for ChipIr) and not always at an exact time interval. The main thing to notice is that the silicon detector shows a better stability, while the diamond suffers by polarization effects that are caused by capture and recombination of charges in defects. These issues are described more extensively by Rebai et al. [14] In these measurements, the effect is more evident for the ChipIr case, probably because the instantaneous flux is higher. Another possible reason is that there is less intensity variability between spills than in CHARM. In fact at ChipIr one can notice about $25 \%$ decrease in count rate over 5 days. At CHARM one can see transient effects lasting a few hundreds of spills (about $1 \mathrm{~h}$ equivalent) after the beam goes down and comes back (see around spill 1000). There are also substantial dips past 6000 number of spills; we can say that this is due to modulation of the intensity of the accelerator, as the traces of the two detectors are in very good agreement.
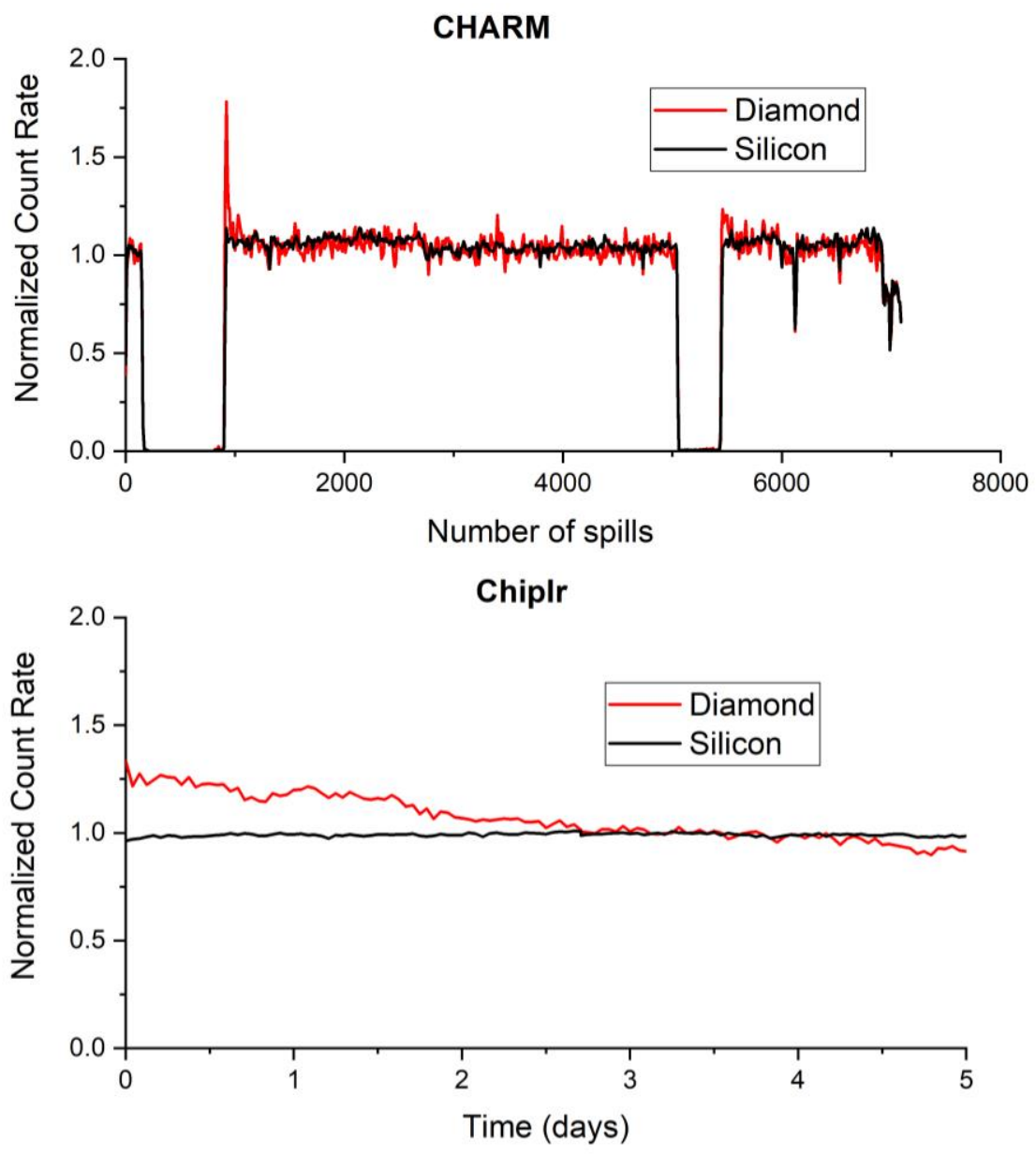

Figure 7. Time traces of the count rates measured at CHARM (top) and ChipIr (bottom) measured with silicon and diamond detectors. The traces are normalized to the average count rate to appreciate more easily the variations with respect to unity. 


\section{Conclusions and outlook}

Silicon and diamond detectors have been used successfully at three spallation facilities. It is shown that a fast digital electronic chain is needed to operate in a pulsed radiation field with high instantaneous flux. At the nTOF facility, fast neutron response functions have been measured using the time of flight technique and results are used to benchmark simulations. At CHARM and ChipIr, these benchmarked simulations are used to predict deposited energy spectra, and the results compared to measurements. Good agreement gives confidence in the experimental characterization and Monte Carlo models of these facilities dedicated to the irradiation of microelectronics.

A study of the stability of these detectors makes the silicon preferable for applications where the total fluences are not too high $\left(<10^{15} \mathrm{n} / \mathrm{cm}^{2}\right)$. In fact, if it is true that diamond is more radiation hard (up to $10^{16} \mathrm{n} / \mathrm{cm}^{2}$ according to the literature), it is shown that polarization effects can compromise the stability and drifts can affect the measurements.

As a continuation of this project, it has been proposed to use $\mathrm{SiC}$ detectors, which are improving in quality and popularity in recent years, and they promise capabilities for higher fluxes and good stability.

\section{References}

[1] M. Rebai, et al. "Pixelated Single-crystal Diamond Detector for fast neutron measurements." Journal of Instrumentation 10.03 (2015): C03016.

[2] C. Zhou, et al. "PIN silicon diode fast neutron detector." Radiation protection dosimetry 117.4 (2005): 365-368.

[3] M. Barbagallo, et al. "Thermal neutron detection using a silicon pad detector and 6LiF removable converters." Review of Scientific Instruments 84.3 (2013): 033503.

[4] B. W. Robertson, et al. "A class of boron-rich solid-state neutron detectors." Applied physics letters 80.19 (2002): $3644-3646$.

[5] M. Rebai et al. "Response of a single-crystal diamond detector to fast neutrons", Journ. of Instrum. 8 P10007 (2013)

[6] H. Pernegger, et al. "Charge-carrier properties in synthetic single-crystal diamond measured with the transient-current technique." Journal of Applied Physics 97.7 (2005): 073704.

[7] C. Weiss, et al., Eur. Phys. J. A., 52, 269, 2016

[8] P. Kavrigin, et al., NIMA, 795, 88-91, 2015

[9] L. Giacomelli, et al. "Diamond detectors for fast neutron irradiation experiments." Nuclear Physics B-Proceedings Supplements 215.1 (2011): 242-246.

[10] A. Pietropaolo, et al. "Single-crystal diamond detector for time-resolved measurements of a pulsed fast-neutron beam." $E P L$ (Europhysics Letters) 92.6 (2011): 68003.

[11] M. Rebai, et al. "Diamond detectors for fast neutron measurements at pulsed spallation sources." Journal of Instrumentation 7.05 (2012): C05015

[12] C. Cazzaniga, et al. "Characterization of the high-energy neutron beam of the PRISMA beamline using a diamond detector." Journal of Instrumentation 11.07 (2016): P07012.

[13] A. Lohstroh, et al. "The effect of fast neutron irradiation on the performance of synthetic single crystal diamond particle detectors." Diamond and Related Materials 19.7-9 (2010): 841-845.

[14] M. Gabrysch, et al. "Electron and hole drift velocity in chemical vapor deposition diamond." Journal of applied physics 109.6 (2011): 063719.

[15] M. Rebai, et al. "Time-stability of a Single-crystal Diamond Detector for fast neutron beam diagnostic under alpha and neutron irradiation." Diamond and Related Materials 61 (2016): 1-6.

[16] C. Cazzaniga, et al. "Charge collection uniformity and irradiation effects of synthetic diamond detectors studied with a proton micro-beam." Nuclear Instruments and Methods in Physics Research Section B: Beam Interactions with Materials and Atoms 405 (2017): 1-10.

[17] E. Normand, IEEE Trans. Nucl. Sci. 43, 2742 (1996).

[18] A. Taber and E. Normand, IEEE Trans. Nucl. Sci. 40, 120 (1993).

[19] C. Frost, et al. "A new dedicated neutron facility for accelerated SEE testing at the ISIS facility." Reliability Physics Symposium, 2009 IEEE International. IEEE, 2009.

[20] C. Borcea, et al. "First results from the neutron facility (nTOF) at CERN." Applied Physics A 74.1 (2002): s55-s57.

[21] C. Cazzaniga and C. D. Frost, "Progress of the Scientific Commissioning of a fast neutron beamline for Chip Irradiation," Journal of Physics: Conference Series, vol. 1021, p. 012037, May 2018. 
[22] J. Mekki et al., "CHARM: A Mixed Field Facility at CERN for Radiation Tests in Ground, Atmospheric, Space and Accelerator Representative Environments," IEEE Trans. Nucl. Sci., vol. 63, no. 4, pp. 2106-2114, Aug. 2016.

[23] B. Riemer, "SEEMS - An SNS-Based Dual Purpose Target Station for Electronics Single Event Effects Testing and Muon Spectroscopy", proceedings of ICANS XXIII, Chattanooga (TN), USA, Oct. 2019

[24] W. Yang, et al. "Atmospheric neutron single event effect test on Xilinx $28 \mathrm{~nm}$ system on chip at CSNS-BL09." Microelectronics Reliability 99 (2019): 119-124.

[25] C. Cazzaniga et al., "Single crystal diamond detector measurements of deuterium-deuterium and deuterium-tritium neutrons in Joint European Torus fusion plasmas." Review of Scientific Instruments, vol. 85, no. 4, p. 043506, Apr. 2014.

[26] A. S., Jacobsen, et al. "Velocity-space sensitivities of neutron emission spectrometers at the tokamaks JET and ASDEX Upgrade in deuterium plasmas." Review of Scientific Instruments 88.7 (2017): 073506.

[27] M. Rebai, et al. "Pixelated Single-crystal Diamond Detector for fast neutron measurements." Journal of Instrumentation 10.03 (2015): C03016.

[28] C. Cazzaniga, et al. "Study of the Deposited Energy Spectra in Silicon by High Energy Neutron and Mixed Fields." IEEE Transactions on Nuclear Science (2019). DOI 10.1109/TNS.2019.2944657

[29] M. Cecchetto, et al. "SEE flux and spectral hardness calibration of neutron spallation and mixed field facilities." IEEE Transactions on Nuclear Science (2019).

[30] D. Chiesa, et al. "Measurement of the neutron flux at spallation sources using multi-foil activation." Nuclear Instruments and Methods in Physics Research Section A: Accelerators, Spectrometers, Detectors and Associated Equipment 902 (2018): 14-24.

[31] C. Borcea, et al. "First results from the neutron facility (nTOF) at CERN." Applied Physics A 74.1 (2002): s55-s57.

[32] E. Aza, et al. "Neutron beam monitoring for time-of-flight facilities with gaseous detectors." Nuclear Instruments and Methods in Physics Research Section A: Accelerators, Spectrometers, Detectors and Associated Equipment 806 (2016): 14-20. 